\title{
Novel Broadband Amplifier for Mid-Infrared Semiconductor laser and applications in spectroscopy
}

\author{
Sheng Wu, Andrei Deev, Yongchun Tang, Zhenyu Li, Axel Scherer \\ PEER Center, California Institute of Technology \\ 738 Arrow Grand Circle, Covina, CA 91722
}

\begin{abstract}
An amplifier design for broadband Mid-IR buried-hetero (BH) structure epitaxial laser is presented, and external cavity design based on this amplifier is described. Spectroscopy results characterizing such single frequency lasers are demonstrated with whispering gallery mode $\mathrm{CaF}_{2}$ disc/ball, saturated absorption in hollow waveguide and direct chemical analysis in water.
\end{abstract}

\section{KEY WORD LIST}

Focused ion beam buried hetero-structure Amplifier, external cavity, Mid Infrared, Whispering Gallery Mode, Quantum Cascade Laser, Saturated absorption, hollow waveguide, and water absorption

\section{INTRODUCTION}

\section{Nano fabricated amplifier for semiconductor epitaxial laser}

A major challenge for building a widely tunable external cavity Mid-Infrared (MIR) laser using latest semiconductor epitaxial (epi) laser is to apply the Anti-Reflection (AR) coating that could turn the otherwise Fabry-Perot (FP) epi laser waveguide into an amplifier. As the epi laser waveguide are progressing rapidly in terms of tuning range [1,2], the AR coating's bandwidth is becoming particularly challenging in the MIR because the change of the wavelength is quite large for a given tuning frequency. For example, as the tuning range of a quantum cascade laser is now reaching $400 \mathrm{~cm}^{-1}$ in the $7-11 \mu \mathrm{m}$ range, the AR coating is impossible to cover such wide range with a residual reflectivity much less than 1\%. In this paper, we present an amplifier design that is nano-fabricated with Focused Ion Beam (FIB) [3]. The amplifier design is universal for semiconductor lasers, particularly useful in the MIR. A single layer broadband AR coating could further improve the performance of this amplifier design by further suppressing the FP modes and reduce the loss of the external cavity (EC) that build upon this amplifier.

In this paper, we tested the tuning range by using this laser to measure the $\mathrm{CO}_{2}$ dissolved in water. Due to strong absorption in the MIR by water, direct spectroscopic measurement of $\mathrm{CO}_{2}$ in water has been limited to optical path lengths of only dozens of microns, and measurable concentration resolution of only $200 \mathrm{mg} / \mathrm{L}$. The high power and brightness of Quantum Cascade lasers could penetrate much deeper optical depths[4-6], i.e. 100s microns, and providing much higher averaging speed to achieve higher concentration resolution. We demonstrate that we could penetrate as deep as 800 micron of water with our tunable EC QC laser built upon the amplifier above.

The linewidth and tuning of the external cavity laser using this amplifier are obtained, and compared with a DFB Quantum Cascade (QC) laser. We used Whispering Gallery Mode (WGM) resonators and Saturated Absorption (SA) spectroscopy of ${ }^{13} \mathrm{CO}_{2}$ and $\mathrm{CDH}_{3}$ in a hollow waveguide to characterize their linewidths.

\section{Linewidths for hetero-structure epi lasers \\ Near-IR DFB and External Cavity diode lasers}

DFB diode lasers are the most mature and stable single frequency laser sources, especially in the Near-IR (NIR) telecom band. However, the inherent high frequency noise in free-running (only constant current and temperature and

Quantum Sensing and Nanophotonic Devices VII, edited by Manijeh Razeghi, Rengarajan Sudharsanan, Gail J. Brown, Proc. of SPIE Vol. 7608, 76080A - (c) 2010 SPIE · CCC code: 0277-786X/10/\$18 · doi: 10.1117/12.841592 
without external frequency locking or stabilization) DFB NIR lasers broadens their Instantaneous Linewidths (ILW, $<100 \mu \mathrm{sec}$ average) to over several MHz. This broadened ILW is actually a merit for DFB lasers working in the telecom, because the broadened ILW will suppress noises generated in the long fiber loop by stimulated Raman scattering and other nonlinear effects.

The past experiments in the NIR with External Cavity (EC) diode laser with a grating as frequency selected feedback suggest that their ILW is much narrower, and $\sim 500 \mathrm{KHz}$ was demonstrated[7] by high precision spectroscopy or high Q cavities, and in telecom more often by Delayed Self-Homodyne RF analysis[8].

The frequency drift, i.e. over msec time scale, of a free running single frequency NIR diode laser under constant temperature $\left( \pm 0.1^{\circ} \mathrm{C}\right)$ and bias current is much larger. This large drift is a result of the varying background temperature causing the internal temperature to drift, and also drift of the current in the laser driver.

\section{Linewidths of DFB and EC Quantum Cascade Lasers}

Free running DFB QCLs are expected to have much narrower ILW than those of conventional Near-IR (NIR) diode lasers operating at the same power. This is because the photon energies are smaller, the cavities are longer $(2 \mathrm{~mm}$ versus $0.2 \mathrm{~mm}$ ) and the linewidth enhancement parameter alpha, or $\alpha$, is expected to be near zero. The first two of these factors reduce the Schawlow-Townes linewidth[9], while the large alpha parameter of NIR diode lasers typically increases the free-running ILW by factors of 10-40 above the Schawlow-Townes limit. It is demonstrated that with low noise drivers, free running DFB QCLs could achieve a linewidth of $150 \mathrm{kHz}$, and the SchawlowTownes linewidth could be much less than $100 \mathrm{kHz}$ for many DFB QCLs[10, 11]. But, detailed spectroscopy evidence was not demonstrated. Frequency stabilization of QCLs further decreases ILW and long term drift to $\mathrm{KHz}$ level and facilitates sub-Doppler spectroscopy, but the stabilization setup which involve external cavity or external saturated absorption is quite complicated[11].

So far, a linewidth of 3.99MHz has been demonstrated for a Mid-IR EC QCL[12], and further stabilization on the EC QCL should prove much finer linewidth like ECL diode lasers stabilized in the NIR. Similar ILW for DFB QC Lasers were reported, and simple external locking has only been able to reduce long term drift and ILW is still several $\mathrm{MHz}[13]$. There is hope that with improved low noise driver, the DFB QCL and EC QCL could achieve linewidths below a couple of $100 \mathrm{kHz}$ without complicated external locking. Here, we investigate the ILW for EC QCL based on the amplifier design above and also for the commercial DFB QCL.

\section{How to measure and characterize the ILW of QC lasers and applications}

So far, the ILW has to be verified by Saturated Absorption (SA) or cavities with ultrahigh Q in the MIR for EC QCLs.

The ultra narrow Doppler free linewidth of stable molecules is a good benchmark gauge for the ILW of QC lasers, be them DFB or EC QC lasers. We could conduct them easily in a compact setup, e.g. Hollow Waveguide (HW). Compared with traditional free space overlapped counter propagating SA setup, the tight overlap is guaranteed in a hollow waveguide as long as the laser could be efficiently coupled into the waveguide. We demonstrated that the coupling efficiency is over 50\% for semiconductor epi lasers, e.g. QC lasers, due to QC laser's high brightness[14]. The transmission loss of $\mathrm{HW}$ is also quite low, only about $1-2 \mathrm{~dB} /$ meter, making it an ideal platform for SA spectroscopy.

To measure the $100 \mathrm{kHz}$ linewidth of a DFB or EC QCLs with a high Q cavity at $5 \mu \mathrm{m}\left(6 \times 10^{13} \mathrm{~Hz}\right)$, the Q should be over $6 \times 10^{8}$, and a whispering gallery mode cavity based on Fluorite crystal discs should be able to realize such $\mathrm{Q}$ easily. 


\section{Applications of Saturated Absorption and WGM in the Mid-IR}

The application of Doppler free ultra-high resolution SA spectroscopy in the MIR had been forecasted ever since its first demonstration[15]. Basically, the Doppler limited spectroscopy has a frequency over resolution of $\sim 6 x 10^{5}$, e.g. at $2,000 \mathrm{~cm}^{-1}$ and Doppler limited resolution of $0.003 \mathrm{~cm}^{-1}$, while the Doppler free SA's spectroscopic resolution, i.e. $100 \mathrm{kHz}$ or $3 \times 10^{-6} \mathrm{~cm}^{-1}$, will lead to nearly 3 orders magnitude increase of information and enable the differentiation of many chemical species previously impossible, e.g. nuclei isomers[16], magnetic hyperfine structures. The major obstacles for SA have been the lack of tunable lasers in the MIR, where the molecules have strong enough absorption to create SA, and the complicated platform of SA spectroscopy. Now, with widely tunable QC and intersubband MIR lasers, and the hollow waveguide, SA could be easily used for real world applications, e.g. the identification of isotopomers of heavy molecules, e.g. UF6, SF6[17], ethane[18], propane.

It have generated lots of interests in the past decade as Whispering Gallery Mode resonators can have ultra-high Q factors over a wide wavelength range in a tiny cavity, which usually could not be realized with conventional cavities with dielectric coatings[ $[19,20]$. The paths of the WGMs lie very close to the surface of the resonator with a portion of the electromagnetic (EM) wave traveling outside, i.e. evanescent wave. Losses due to absorption or scattering of the evanescent wave outside the resonator will change the $\mathrm{Q}$ factor of the resonator. Several groups demonstrated that WGM resonators could be used for absorption sensing in gases and liquids, detection of refractive index changes around the resonator and single molecules on its surface[21, 22]. All chemical sensing studies performed until now with WGM used near-IR and visible continuous wave (CW) single frequency lasers because of the wide availability of such lasers and detectors. However, mid-Infrared (MIR) and far-Infrared (FIR) regions of the spectrum are much more important for chemical sensing because the fundamental bands of vibrations lying in these regions are 1 3 orders of magnitude stronger than their near-infrared overtones. However, there are some difficulties in realizing MIR/FIR coupled WGMs., i.e. there are very few choice of single mode fibers in the MIR/FIR, and the most common fused silica material is not transparent in that range, and finally CW QCL sources are still far from as popular as telecom CW diode lasers, but the latest improvements in QCLs bode well for their applications.

Because of the high brightness of QCLs, we recently demonstrated that the radiation of a QC laser could be efficiently coupled into a $300 \mu \mathrm{m}$ diameter hollow waveguide and used as a microliter gas sensor in gas chromatography. We also demonstrate that pulsed QCLs, in spite of their relatively large linewidth, could be efficiently coupled into WGM cavities.

In this paper, we demonstrate, to the best of our knowledge, the first SA spectroscopy of molecules in a hollow waveguide with unprecedented overlap and saturated intensity. Also, we demonstrate, the first observation of WGM spectra with Q well over 1 million in the MIR excited with a CW laser.

\section{EXPERIMENT}

\section{Amplifier fabricated with Focused Ion Beam}

The schematic of the amplifier build upon a semiconductor epi is given in Figure series 1. The material of the wafer is kept as short as possible to reduce the free carrier absorption in the MIR[23-25]. The fabrication is carried out with the aid of XeF, which help to clean the residuals of the FIBs material. This proves to be a significant step, because withput the cleaning, the resistance of the QC epi is very low, and the laser will burn at the FIB cutting positions once powered up.

\section{Water penetration and $\mathrm{CO}_{2}$ measurement}

We setup the water penetration test as shown in figure $2 \mathrm{~d}$. We also flow pure $\mathrm{CO} 2$ in the open air beam path to get gas phase $\mathrm{CO} 2$ absorptin features in our scan, see figure $2 \mathrm{~b}$. We are able to observe reasonable signal to noise ratio scans even when water depth is over $500 \mu \mathrm{m}$. 


\section{WGM observation with EC and DFB QCL}

The setup is similar to the experiment where we demonstrated the pulsed QCL coupling into WGM reported last year. The detector has a bandwidth of $20 \mathrm{MHz}$, and the scan rate of the DFB laser has to be slow enough to let the detector resolve the sharp features of the WGM spectra. But at slow scanning rate, the jitter of the DFB QC laser will become the major limiting factor. The free running DFB QC lasers have been demonstrated to have an estimated bandwidth of over $10 \mathrm{MHz}$ at $1 \mu \mathrm{sec}$ scale. Therefore, the observable $\mathrm{Q}$ at $2,280 \mathrm{~cm}^{-1}$ will be limited to $8 \times 10^{6}$.

We carefully adjusted the angle of incidence (AOI) of the QCL beam at the hypotenuse face so that only the lowest order WG modes, i.e. $q=1$, are excited. We achieved near single mode excitation for $q=1 \mathrm{WG}$ modes when we adjust the AOI of QCL at the hypotenuse to be slightly above the critical angle for total internal reflection to happen at the $\mathrm{ZnSe}$ and $\mathrm{CaF}_{2}$ interface, which is $35.3^{\circ}$ at $4.5 \mu \mathrm{m}$ wavelength. As AOI getting closer to this critical angle, the WGM structure starts to simplify.

We obtained $\mathrm{CaF}_{2}$ discs by OEwaves using procedures for fabricating high $\mathrm{Q} \mathrm{CaF}_{2}$ WGMs[26], we also fabricated $\mathrm{CaF}_{2}$ and $\mathrm{BaF}_{2}$ discs with different morphology on the outskirt. Results of these high Q WGM resonators will be obtained in the coming weeks.

\section{Saturated Absorption in Hollow Waveguide with EC and DFB QCL}

We coupled laser output from our EC and DFB QCL into the $300 \mu \mathrm{m}$ ID HW, and achieved $>50 \%$ coupling and over $40 \%$ output after 0.5 meter HW length. The laser output from the other side of the HW is back reflected with a concave gold coated reflector, matching the Numeric aperture of the HW. The back-reflected signal is sampled by a $\mathrm{ZnS}$ beam splitter and detected by a MCT detector. The $\mathrm{ZnS}$ beam splitter has a Fresnel reflectivity of $>20 \%$ at each surface at $45^{\circ}$ Angle of incidence, and therefore a total of $4 \%$ each face of power is reflected into the detector after double passing the HW.

\section{RESULTS}

Figure series 2 show the water absorption spectra in the MIR, and the optical pathlength is severely limited by the strong MIR absorption by water to 10s microns for FTIR instrument. With QC lasers, we demonstrated that could have reasonable signal to noise ratio of 4:1 even when the water depth is over $500 \mu \mathrm{m}$.

Figure series 3 show the WGM spectra. A maximum WGM coupling induced loss of $10 \%$ is observed when optimized. The period spacing between the WGM spectra is measured to be $\sim 0.45 \mathrm{~cm}^{-1}$, which is calibrated with an air spaced Ge etalon with a Free Spectral Range of $0.075 \mathrm{~cm}^{-1}$. This value is consistent with the size of the $\mathrm{CaF}_{2}$ ball lens, i.e. $5 \mathrm{~mm}$ diameter. In fact, we noticed that the WGM modes for this $5 \mathrm{~mm} \mathrm{CaF}_{2}$ ball are much simpler at $4.46 \mu \mathrm{m}$, when compared to the case at $1.55 \mu \mathrm{m}$, i.e. with the same setup at the critical AOI for $1.55 \mu \mathrm{m}$ as shown in the insert of figure 2. We attribute this reduction in the number of WGM dips to the facts that we are using a $4.46 \mu \mathrm{m}$ laser, instead of a $1.5 \mu \mathrm{m}$ laser. The longer wavelength laser reduces the equator's WGMs to almost single $q=1$ operation under the critical AOI coupling. The measured Q is over $2 \times 10^{6}$ in the MIR of $2,280 \mathrm{~cm}^{-1}$.

\section{DISCUSSIONS}

\section{Amplifier for widely tunable QC lasers}

The amplifier design presented here had already been demonstrated before with telecom diode epi lasers. However, the free carrier absorption at the telecom band is negligible whereas it is quite strong in the MIR. It is not a must to use highly doped wafer for QC epi growth, and in fact low doped wafers have been routinely demonstrated with high performance QC lasers. However, it is still crucial to keep the wafer material in the amplifier design as short as possible to minimize the free carrier absorption loss. 
It has also been demonstrated that a broadband AR coating could further reduce the modulation of the residual FP cavity of the epi[3]. Such coating could achieve $<5 \%$ reflectivity over the entire MIR range from $<3 \mu \mathrm{m}$ to $>10 \mu \mathrm{m}$.

\section{Measuring $\mathrm{CO} 2$ in water}

The absorption by water in the MIR is the major limiting factor for any chemical analysis by optical absorption spectroscopy. Traditional FTIR spectroscopy setups only have 10s of microns optical depths. Here, the QC lasers have high power output concentrated in a narrow bandwidth, the brightness is several orders of magnitude higher than FTIR, and the repetition average is much higher as well. We hope to apply this technique for the Monitoring, Verification and Accounting (MVA) of underground brine $\mathrm{CO} 2$ sequestration. We expect to achieve a resolution of $0.2 \mathrm{mg} / \mathrm{L}$.

\section{Saturated absorption inside hollow waveguide}

The currently demonstrated SA linewidth of $10 \mathrm{MHz}$ is mainly limited by the laser stability. If a stabilized laser, i.e. driven with low noise drive, the laser linewidth should be close to $100 \mathrm{kHz}$. Then, we could realized much higher resolution with SA in the HW.

The linewidth of the Saturated Absorption for stable molecules, e.g. $\mathrm{CO}_{2}$, is determined by several factors in the waveguide. The first is the transit time of the molecules inside the optical field due to the relatively small diameter of the HW. The transit time induced linewidth is calculated to be roughly following this equation,

$\mathrm{D}_{\mathrm{wall}} * \Delta \mathrm{v}_{\mathrm{tr}} \sim 100 \mathrm{kHz} * \mathrm{~mm}$,

where $\mathrm{D}_{\text {wall }}$ is the dimension of the hollow waveguide, i.e. $350 \mu \mathrm{m}$, and $\Delta v_{\mathrm{tr}}$ is the transit time limited linewidth will be $300 \mathrm{kHz}$. At a pressure of $\sim 0.05 \mathrm{Torr}$, the pressure broadening $\left(\sim 4 \mathrm{MHz} /\right.$ Torr for $\left.\mathrm{CO}_{2}\right)$ will be $200 \mathrm{kHz}---$ less than transit time broadening. This means that the hollow waveguide could be coupled with GC and vacuum interfaced to provide ultra-high resolution spectroscopy analysis, if column switch is used to trap individual chemical peaks inside a long HW for analysis.

\section{WGM spectra in $\mathrm{CaF}_{2}$ ball/disc}

Since the energy loss in the ball is dominated by scattering loss, and Q is therefore proportional to the cube of the wavelength, as given in equation[27]:

$$
Q \approx \frac{3 \lambda^{3} a}{8 n \pi^{2} B^{2} \sigma^{2}}
$$

where $\lambda$ is the wavelength, $a$ is the radius of the WGM cavity, $n$ is the refraction index of the resonator material, B is the correlation length, and $\sigma$ is the roughness. For the same $\mathrm{CaF}_{2}$ ball, we expect to observe $\sim 23$ times higher $\mathrm{Q}$ at $2,280 \mathrm{~cm}^{-1}$ than at $6,450 \mathrm{~cm}^{-1}$. Instead, we only observed roughly 4 times higher $\mathrm{Q}$. The missing $\mathrm{Q}$ could be obscured by the limited resolution of our laser. We expect that our EC QCL laser could have narrower linewidth, i.e. 100kHz, if a low noise driver could be used.

As demonstrated with fluorite crystalline WGM cavities in the near IR, a $\mathrm{CaF}_{2} \mathrm{WGM}$ resonator could potentially achieve values over $10^{9}$ for the Q factor at $1.5 \mu \mathrm{m}$ after careful polishing and annealing. Because of the longer wavelengths in the MIR and FIR, the main Q limiting factor will no longer be the surface scattering loss but the material absorption. This lower requirement on surface quality will make WGM sensors more robust in the MIR/FIR, i.e. they would be less sensitive to dust and environmental changes. $\mathrm{CaF}_{2}$ already starts to have higher absorption at the wavelengths over $4 \mu \mathrm{m}$ and limit its $\mathrm{Q}$ at $4.5 \mu \mathrm{m}$ to below $10^{9}$, while $\mathrm{BaF}_{2}$ could be a better candidate if we need to use longer IR wavelengths or get even higher $\mathrm{Q}$ factors at $4.5 \mu \mathrm{m}$. 
Although fluorite crystalline WGMs resonators potentially have very high Qs in the MIR/FIR, this does not translate into very long effective path length for chemical sensing, especially if we try to sense gas phase chemicals. This is because the evanescent wave only accounts for a small portion of the Electro Magnetic (EM) field of the WGMs, and only the evanescent wave interacts with external chemicals. The fraction of the WGM's energy in the evanescent field depends on the refractive indices inside and outside resonator. For gas phase sensing, this fraction, $f$, is only $\sim 1 \%$ or less[22]; while for liquid phase, $f$ could be as high as $30 \%[21]$. Therefore, with the $\mathrm{Q}$ value of $>10^{8}$ in the gas phase and $>10^{7}$ in the liquid phase, the effective sensor path length would exceed 1 meter. One meter path length would be sufficient for liquid sensing but in the gas phase it is much shorter of the path lengths provided by multipass cells (1 >100 meters) and cavity enhanced methods ( $>1,000$ meters). However, WGM sensors could be used in applications that require small sample volume or overall small size of the sensors. For example, they could be used as inline sensor for Gas Chromatography (GC). With a one meter path length one could detect ppbV concentrations of $\mathrm{CO}_{2}$, which would exceed the sensitivity of the mainstream GC sensors.

\section{CONCLUSION}

We report the design and fabrication of an epi level amplifier for Mid-IR lasers. The applications of widely tunable single frequency laser, i.e. measuring chemicals in water, and coupling with the hollow waveguide and Whispering Gallery Mode cavity and use as sensors for chemicals are demonstrated and discussed.

We gratefully acknowledge the helpful discussions on WGMs with Dr. Ivan Grudinin, Dr. Andrey Matsko and Dr. Vladmir Ilchenko. We are in debt to Prof. Axel Scherer for his generous hours given us on the use of the KNI nano fabrication facility and insightful guidance on FIB fabrication. 


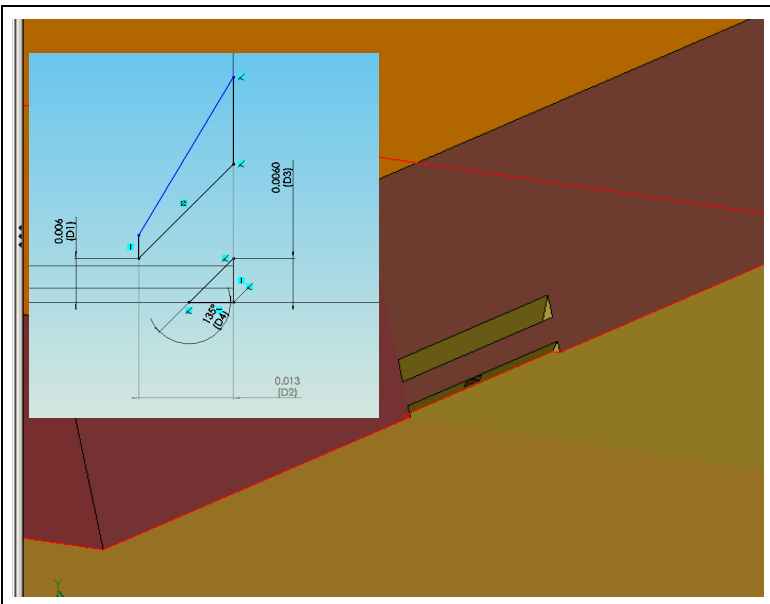

Fig. 1a. Schematic of the FIB cut on the output of the epi waveguide to form an amplifier

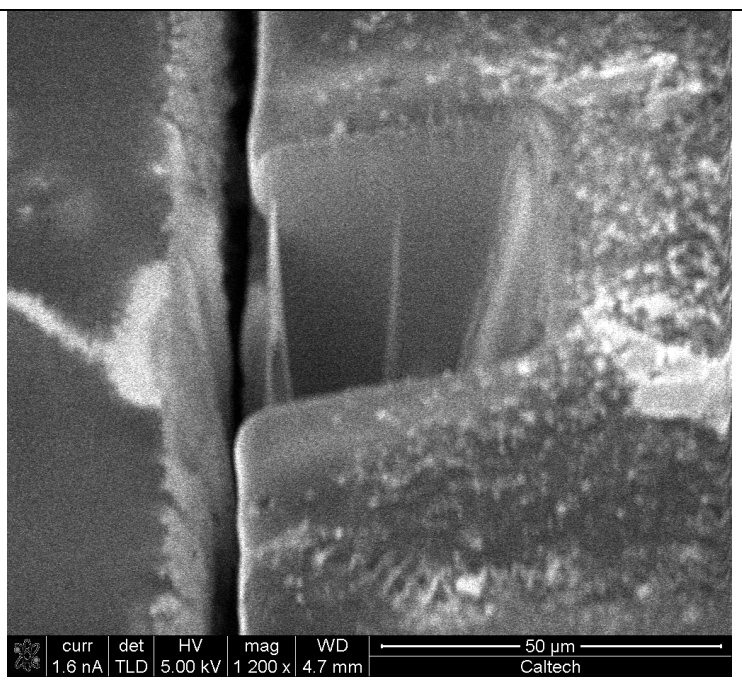

Fig. 1b. Preliminary FIBs cut on the QCL epi output 


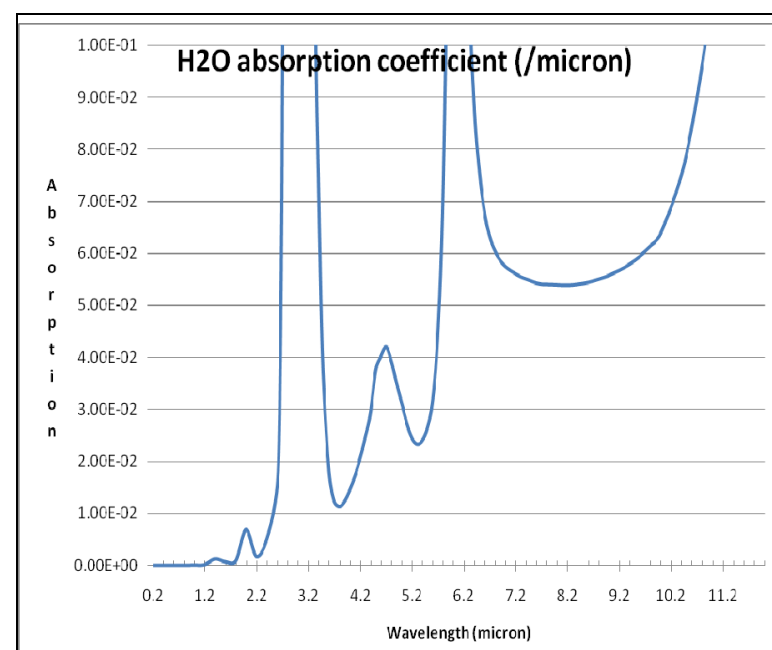

Fig. 2a. Water absorption from UV to MIR. The absorption is very strong when wavelength $>2 \mu \mathrm{m}$

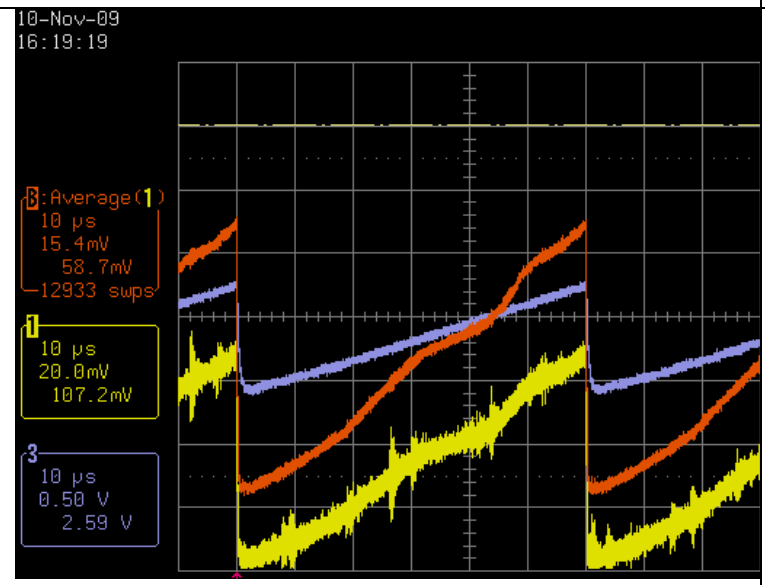

Fig. 2c. Scan of the QC laser at 2,280 cm-1, and the optical depth of water is $500 \mu \mathrm{m}$, noise flow is about $5 \mathrm{mV}$.

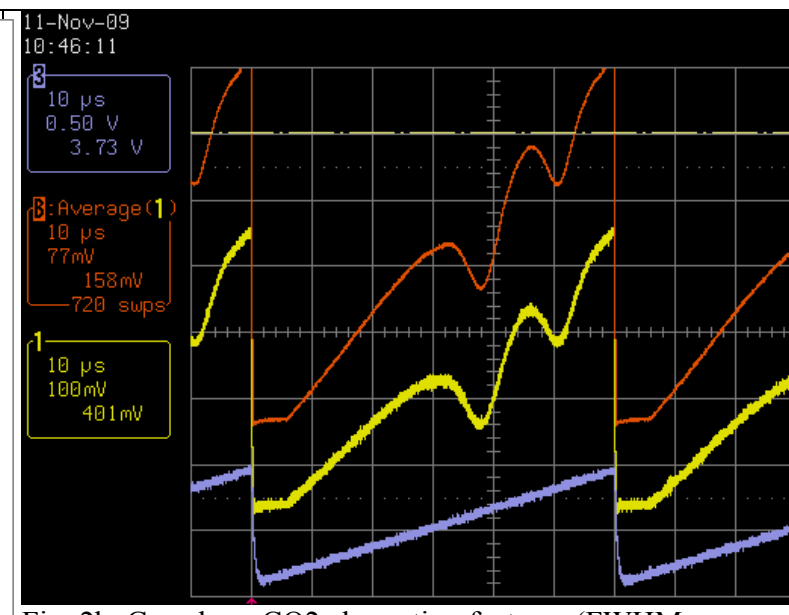

Fig. 2b. Gas phase CO2 absorption features (FWHM $\left.0.1 \mathrm{~cm}^{-1}\right)$ are visible along the scan which goes through $100 \mu \mathrm{m}$ water, high concentration of $\mathrm{CO} 2$ in the atmosphere is created by flow of pure $\mathrm{CO} 2$ in the air.

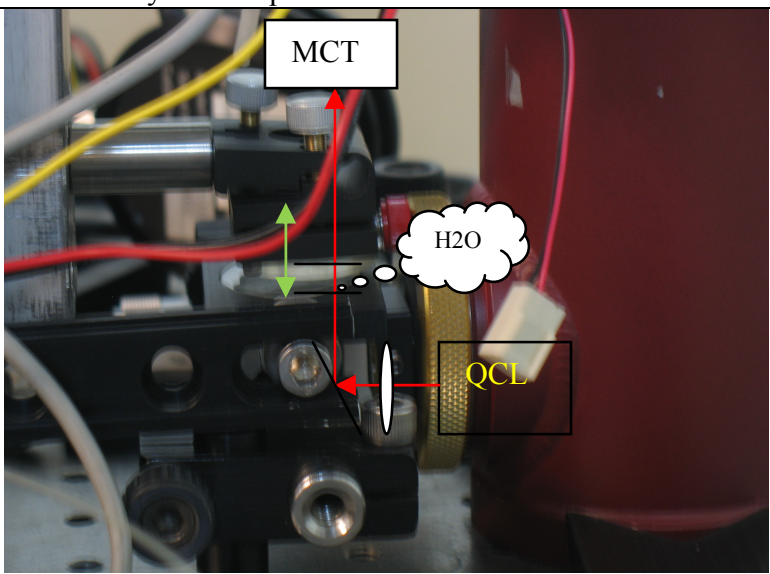

Fig. 2d. Experimental setup of the water penetration and measurement of $\mathrm{CO} 2$ dissolved in water. Test laser is a $\mathrm{CW}$ cryogenic cool QC laser with power of $30 \mathrm{~mW}$. 


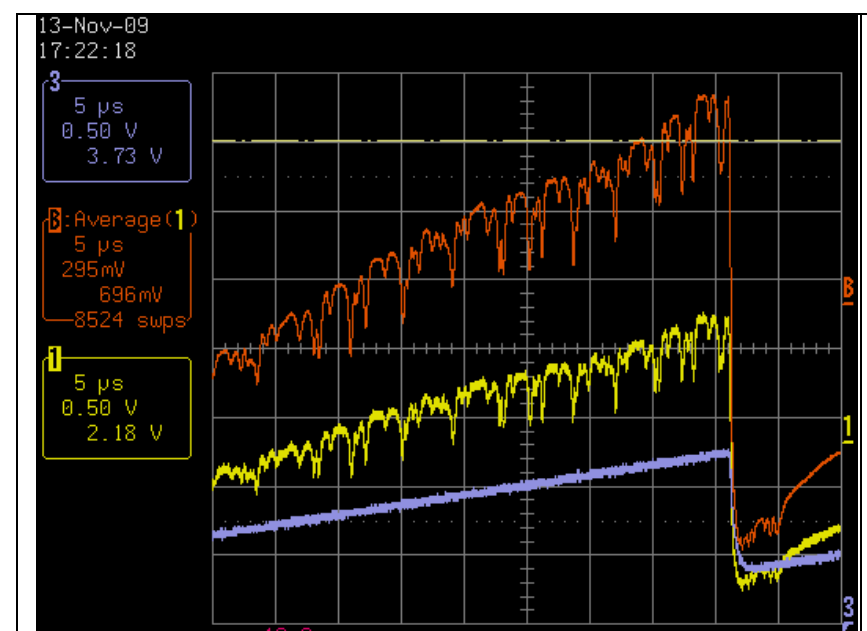

Fig. 3a. Coupling angle smaller than critical angle from $\mathrm{ZnSe}$ to

$\mathrm{CaF}_{2}$

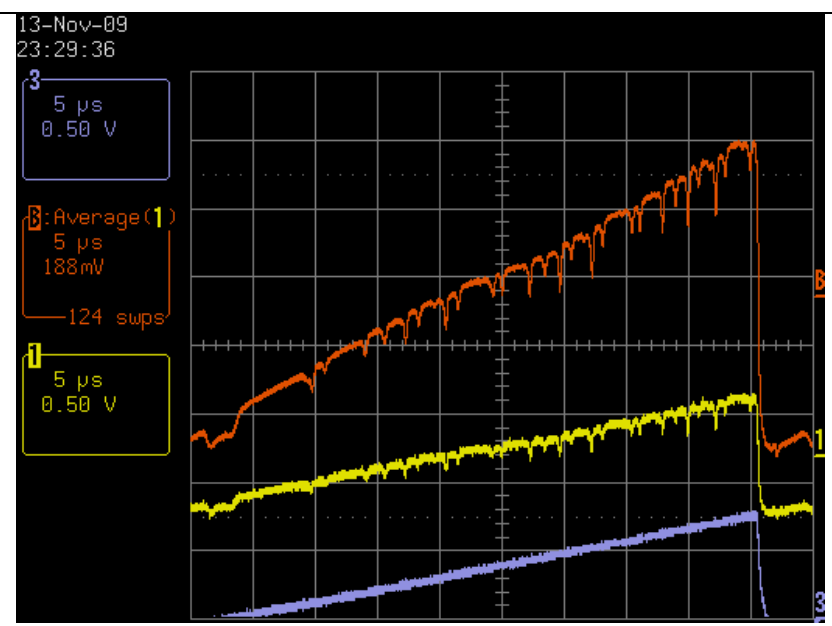

Fig. 3c. Coupling angle $\sim$ critical angle from $\mathrm{ZbSe}$ to $\mathrm{CaF}_{2}$, resulting much reduced simplified spectra

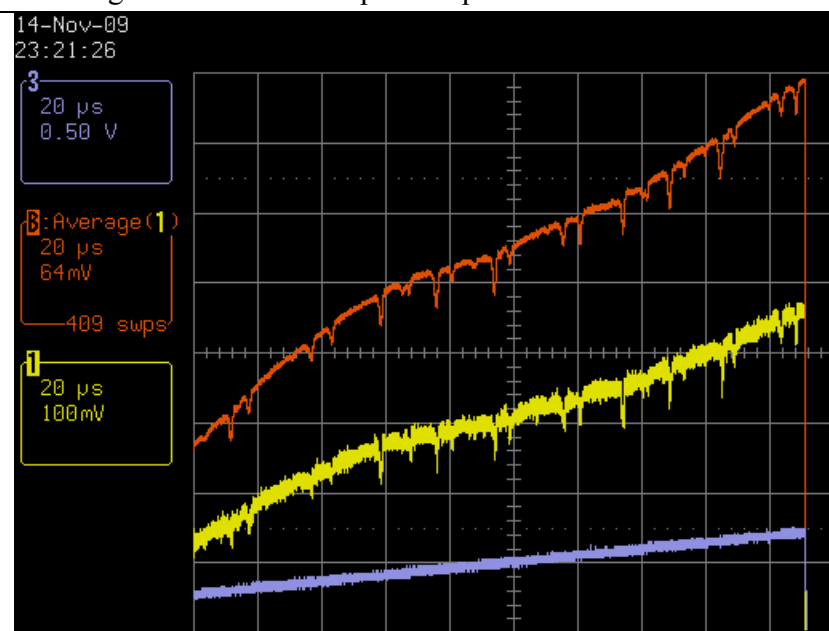

Fig. 3e. WGM spectra with period about $75 \mu \mathrm{sec}$ or $0.47 \mathrm{~cm}-1$ FSR

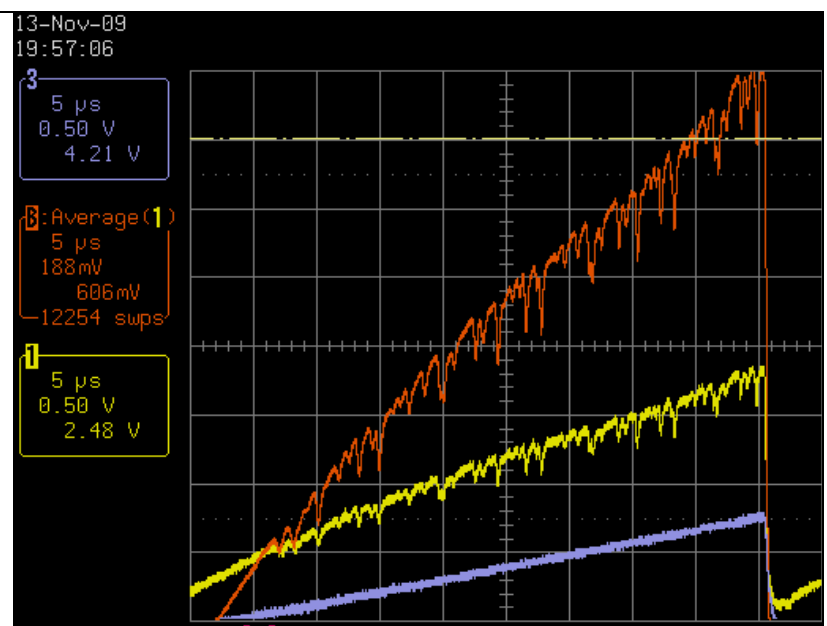

Fig. 3b. Coupling angle closer to critical angle from $\mathrm{ZnSe}$ to $\mathrm{CaF} 2$

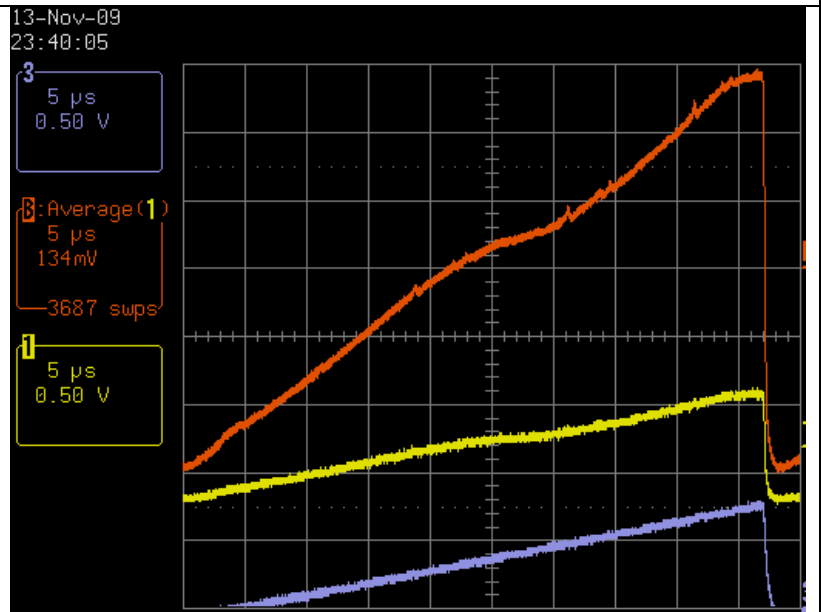

Fig. 3d. Coupling angle $>$ critical angle from ZS to CF, only reversed spectra are observed

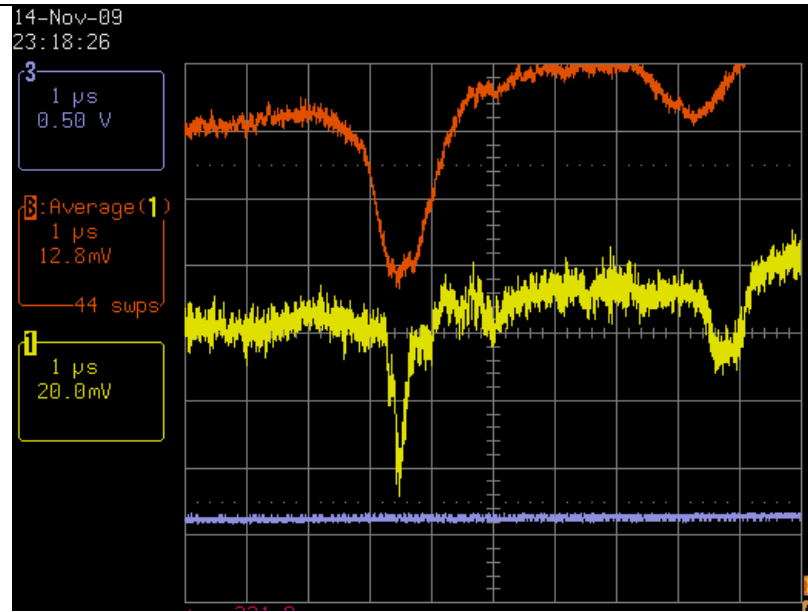

Fig. 3f. Zoom-in single scan (below) FWHM of $<0.2 \mu \mathrm{Sec}$, or $\sim 0.001 \mathrm{~cm}^{-1}$. The Q should be larger than $2 \times 10^{6}$ at $2,280 \mathrm{~cm}^{-1}$. The averaged scan (upper) has much larger linewidth due to 


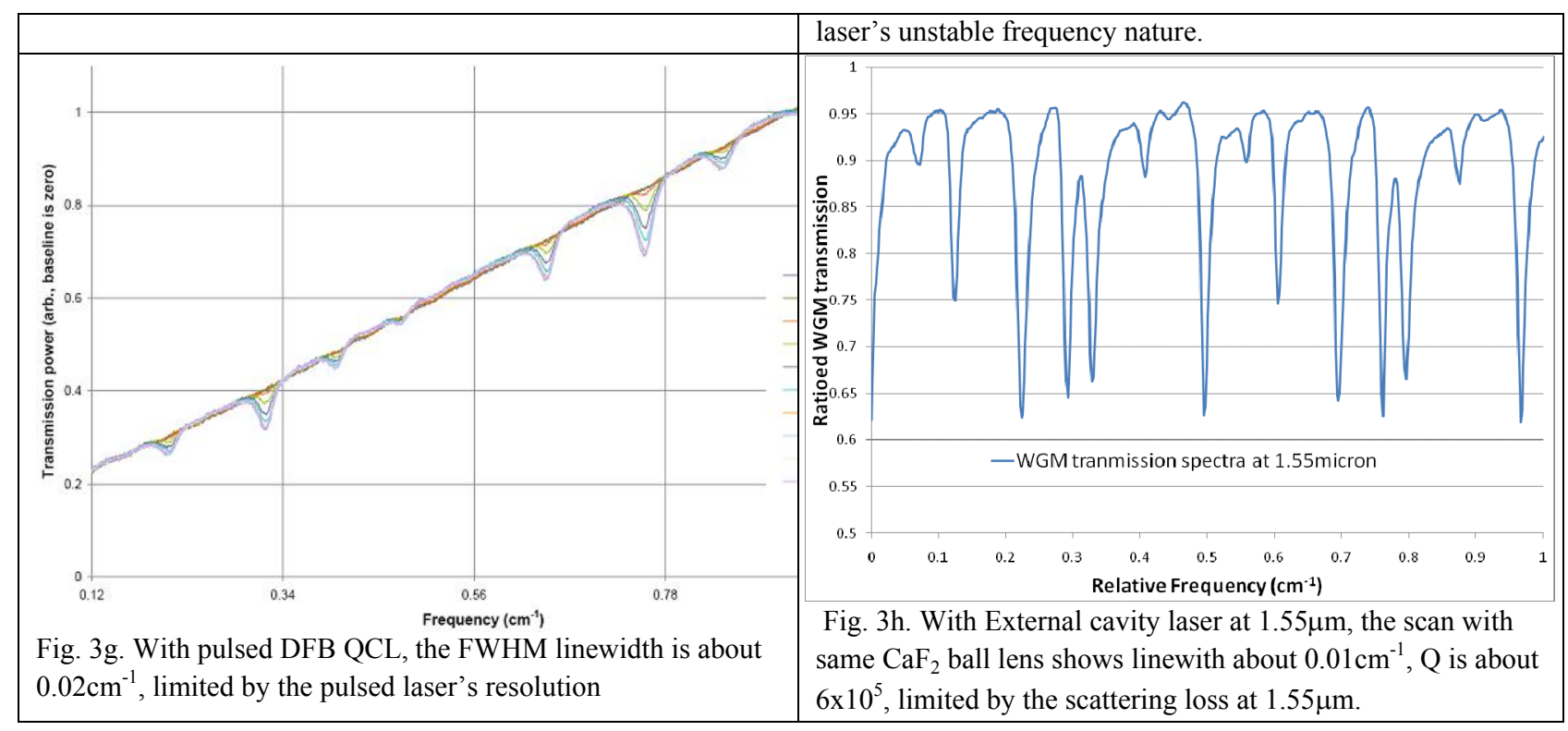

Proc. of SPIE Vol. 7608 76080A-10 